\title{
Hedgehog signaling dynamics in mouse embryos determined by a bioluminiscent reporter
}

\author{
DAVID HERNÁNDEZ-GARCÍA\#, DANIEL FUENTES-JIMÉNEZ\#, VERÓNICA ROJO-LEÓN, CHRISTOPHER WOOD*, \\ CELINA GARCÍA* and LUIS COVARRUBIAS* \\ Departamento de Genética del Desarrollo y Fisiología Molecular, Instituto de Biotecnología, \\ Universidad Nacional Autónoma de México, Morelos, México
}

\begin{abstract}
Determination of cellular signaling in live embryos is key to understand the molecular processes that drive development. Here, we show that a transgenic mouse line carrying a luciferasebased gene reporter of Gli-mediated transcriptional activation (Gli-Luc) displays sonic hedgehog (Shh) signaling in discrete developmental processes during short-term cultures of whole embryos or embryo explants. The bioluminescence in E9.5 embryos was detected in regions in which Shh activity has been demonstrated. Later, in E10.5 embryos, bioluminescence intensity markedly increased, mostly corresponding to the high Shh activity of the developing midbrain and limb. Notably, the dynamic range of the Gli-Luc reporter in the developing limb revealed the progressive emergence of bioluminescence in the zone of polarizing activity, where reporter activity locally increased and spatially spread in agreement with the signaling gradient expected for Shh. In the midbrain of E9.5 mouse embryos, bioluminescence was not detected along the ventral region as expected but, instead, Shh-dependent anterior and posterior bioluminescence foci emerged by E10.5 indicating that the Gli-Luc reporter can only respond transcriptionally to relatively high levels of GliA and/or without the interaction with other transcription factors. The present work supports the use of bioluminescence to identify and study the dynamics of centers of morphogen signaling during mouse embryogenesis.
\end{abstract}

KEY WORDS: sonic hedgehog, luciferase, embryogenesis, midbrain, limbs

\section{Introduction}

Cell signaling is a constant and dynamic process that is behind all molecular mechanisms that control cell behavior during development. Cell signaling involves the activation of a receptor and its transduction into changes in the concentration of second messengers and/or in activation of signaling protein phosphorylation cascades that may ultimately end in modifications of gene expression due to a single or few transcription factors. Therefore, the activity of cell signaling pathways in time windows of minutes or hours can be deduced from the levels of activity of their associated transcription factors. This can be achieved by measuring the expression levels of a reporter transgene that carries one or several copies of the specific transcription factor binding sequence fused to a basal promoter.
Bioluminescent reporter assays are convenient tools for the detection and measurement of different aspects of biological functions, including transcriptional activity in live organisms (Welsh \& Kay 2005; Saito et al., 2012; Iwano et al., 2018). One reporter protein frequently employed in these assays is the luciferase enzyme of the firefly Photinus pyralis which, in the presence of its substrate luciferin, produces photons. By employing highly sensitive detectors coupled to micro- and macro-scopes, spatially resolved gene expression levels can be followed dynamically over several hours, due to the assay's low toxicity, wide dynamic range and fast response to changes in transcriptional activity (Thorne et al., 2010). Assays with this or similar luciferases have been

Abbreviations used in this paper: Gli-Luc: luciferase-based gene reporter of Gli-mediated transcriptional activation; Shh, sonic hedgehog; ZPA, zone of polarizing activity.

\footnotetext{
*Address correspondence to: Luis Covarrubias / Celina García / Christopher Wood. Av. Universidad 2001, Cuernavaca, Morelos, 62210, México. Tel: +52 (777) 3290817 / +52 (777) 3291636. E-mail: covs@ibt.unam.mx / celina@ibt.unam.mx / chris@ibt.unam.mx - web: http://www.ibt.unam.mx (iD) https://orcid.org/0000-0002-4066-4915 (LC); iD https://orcid.org/0000-0003-3433-0115 (CG) and (iD) https://orcid.org/0000-0001-7175-7121 (CW)
}

\#Note: Both authors contributed equally to this study

Supplementary Material (9 supplementary videos for this paper is available at: https://doi.org/10.1387/ijdb.190098LC)

Submitted: 5 June, 2019; Accepted: 16 October, 2019.

ISSN: Online 1696-3547, Print 0214-6282 
implemented for visualization of live cell cultures and live whole organisms (Welsh \& Kay 2005; Saito et al., 2012; Iwano et al., 2018). Fluorescent proteins are alternative non-invasive reporters of transcriptional activity, but despite significant improvements and increased diversity, luciferases retain advantages where phototoxicity must be minimized (e.g., long-term imaging), high sensitivity is required, or where the use of most fluorescent proteins to measure gene activity within whole complex organisms (e.g., mammals) is limited by the intrinsic opacity and autofluorescence of most tissues (Saito et al., 2012).

In contrast with zebrafish, the entire process of mouse embryogenesis cannot be observed in real time. Conveniently, explant cultures of pieces of embryo or organs are alternative options to study mouse developmental processes in real time. This type of experimental approach closely reproduces cellular and molecular events associated with a specific developmental process within a determined time window, and has been validated for, among others, both embryonic midbrain and limb development (Baizabal, Covarrubias, 2009; Hernández-Martínez et al., 2009). Therefore, determination of real time transcription dynamics related to specific cell signaling pathways in explant cultures could provide valuable information to understand cell communication during mammalian development.

The Hedgehog $(\mathrm{Hh})$ signaling pathway plays a major role in many developmental processes. Among these, the morphogenetic activity of Sonic hedgehog (Shh) has been studied in detail in two contrasting functions: the specification of neural precursors within the neural tube (Blaess et al., 2011; Ribes et al., 2010) and the specification of digit identity in the developing limb (Ahn, Joyner, 2004; Harfe et al., 2004). In mammals, Shh mediates its effect through the regulation of three Gli family members (Gli13 ), which can activate (GliA; mainly Gli1 and Gli2) or repress transcription (GliR; mostly Gli3) by binding to the Gli binding sequence (GBS; Jiang, Hui, 2008). Here, we used whole embryo and explant cultures derived from a transgenic mouse line that reports transcriptional activation by GliA, to observe the dynamics of Shh activity in different developmental contexts.

\section{Experimental Protocols}

\section{Animals}

All animal protocols were approved by the Bioethics Committee of the Instituto de Biotecnología-UNAM and performed in accordance with its guidelines. The mice were housed in the animal facility of the Instituto de Biotecnología-UNAM on a $12 \mathrm{~h}$ light/ dark diurnal cycle. Food was provided ad libitum. The embryos employed in the assays were derived from intercrosses among Fvb/NJ Tg(Gli-Luc) homozygous mice or between homozygous for the transgene and Fvb/NJ wild type mice to obtain hemizygous mice. Embryos hemizygous for the transgene were used for all determinations, except for the ones done with midbrain explants (see below). The Gli-Luc transgene consists of eight tandem GBS repeats in proximity to the $\delta$-crystallin basal promoter upstream of the Photinus pyralis luciferase coding sequence (Kimura et al., 2008). Parents of embryos were genotyped using specific primers in the Gli-Luc transgene sequence (forward, 5'CGGGCGCGGTCGGTAAAGT3'; reverse, 5'AACAACAACGGCGGCGGGAAGT3') and/or by determining bioluminescence intensity in ear pieces.

\section{Whole embryo and embryo explant cultures}

E9.5-E11.5 embryos (i.e., embryos at 9.5-11.5 days post coitum; 0.5 was the day of plug detection) were put on a layer of $0.8 \%$ agarose in PBS supplemented with $1 \mathrm{mM} \mathrm{D-Luciferin} \mathrm{(Gold} \mathrm{Biotechnol-}$ ogy, St. Louis, MO) in a $35 \mathrm{~mm}$ Petri dish (Corning Incorporated, Corning, NY) covered with $1 \mathrm{ml}$ of KSOM medium (Embryomax; Millipore, Kenilworth, NJ); cultures were for up to $27 \mathrm{~h}$. In the case of E9.5 and E10.5 embryos, we used $50 \mu \mathrm{M}$ of cyclopamine (CYC) (Sigma-Aldrich, St. Louis, MO) and DMSO drug vehicle was used for control cultures. The explant culture procedure was based on the protocol previously described (Baizabal, Covarrubias, 2009; Fig. 1A). For real-time observations, a group of four explants were placed on a $35 \mathrm{~mm}$ cover-glass bottom Petri dish (World Precision Instruments, Sarasota, FL) with the ventricular surface facing upwards and individually embedded in a collagen matrix. For treatment with a SMO inhibitor (200 nM SANT-1; Sigma-Aldrich, St. Louis, MO), individual explants were placed in one of a 4-chamber $35 \mathrm{~mm}$ glass bottom Petri dish (In vitro Scientific, Mountain View, CA); SANT-1 was added $3 \mathrm{~h}$ after the addition of media supplemented with DLuciferin and cultured for an additional $12 \mathrm{~h}$. Cultures of limbs were performed as previously described (Hernández-Martínez et al., 2009). Briefly, dissected limbs of E10.5 embryos were placed on a $0.22 \mu \mathrm{m}$ Durapore GVPP membrane (Millipore, Kenilworth, NJ) which was let floating on DMEM medium with a drop of it added to each sample in a 4-chamber $35 \mathrm{~mm}$ glass bottomed Petri dishes with a SMO agonist (100-200 nM SAG; Santa Cruz Biotechnology, Inc, Dallas, TX) or a SMO antagonist (50 $\mu \mathrm{M} \mathrm{CYC)} \mathrm{added} \mathrm{to} \mathrm{sepa-}$ rate chambers and incubated in a $5 \% \mathrm{CO} 2$ incubator at $37^{\circ} \mathrm{C}$ for $6 \mathrm{~h}$. After culture, limbs were incubated in $1 \mathrm{ml}$ of KSOM medium containing $100 \mathrm{nM} \mathrm{D}$-Luciferin for $10 \mathrm{~min}$ in a 5\% CO2 incubator at $37^{\circ} \mathrm{C}$. Bioluminescence images of midbrain explants or limbs after culture were acquired either with a $2 \mathrm{X}$ objective as described below or within the In-Vivo Xtreme (Bruker, Billerica, MA) with a 7.2 $\mathrm{cm}^{2}$ field of view, using a binning factor of $4 \times 4$, aperture f/stop of 1 , and an acquisition time of $3 \mathrm{~min}$, with no emission filter in place.

\section{Real time bioluminescence assay procedure}

For real time determination of luciferase activity, the Petri dish with embryos or midbrain explants was placed into an incubator chamber (INU ZILCS F-1 Tokai Hit, Fujinomiya-shi, Shizuoka, Japan) located on the stage of a macroscope (MVX10 Macroview; Olympus Shinjuku, Tokyo, Japan) (Fig. 1B). The parameters for the incubator chamber were $41^{\circ} \mathrm{C}$ for top heater and $38^{\circ} \mathrm{C}$ for the stage heater. $\mathrm{CO}_{2}$ was pumped at $5 \%$ in humidified air to the chamber by a dosing pump ( $\mathrm{CO}_{2}$ Module $\mathrm{S}$ and Humidifier S; Pecon, Erbach, Germany). No emission filters were employed in the detection light path. For imaging of explants, bright-field and luminescence images were acquired with an EM-CCD camera device (iXon X3897; Andor, Belfast, UK) attached to the macroscope top port. For luminescence acquisition, water cooled to $10^{\circ} \mathrm{C}$ was circulated (Oasis 160; Solid State, Wappingers Falls, NY) through the cooling chamber of the camera. The camera device was controlled by computer using Micromanager 1.4 (https://www.micro-manager.org/), with the Highest Dynamic Range (Conventional Amplifier) acquisition mode at $10^{\circ} \mathrm{C}$ for bright-field images and Photon Counting for Large Acquisition Time ( $>1 \mathrm{~s}$ ) mode at $-90^{\circ} \mathrm{C}$ for luminescence images. One brightfield image was taken at the beginning of the experiment and then a time-lapse image acquisition program proceeded to work for up to $27 \mathrm{~h}$, with one 10-min exposure image taken every 11 minutes 
without on chip binning (effective pixel size $16 u \mathrm{~m} \times 16 u \mathrm{~m}$ ).

For imaging of whole embryos, bright-field and luminescence images were acquired with a slow scan CCD camera device (iKon $\mathrm{M}$; Andor, Belfast, UK) attached to the macroscope top port, with water-cooling as described for explant imaging. The camera device was controlled using Micromanager 1.4 (https://www.micro-manager. org/), with bright-field images acquired at $10^{\circ} \mathrm{C}$ chip temperature (100-500 ms exposure), and luminescence images acquired at chip temperature of $-95^{\circ} \mathrm{C}, 0.05 \mathrm{MHz}$ read-out. One bright-field image was taken at the beginning of the experiment and then a time-lapse image acquisition program was applied for up to $27 \mathrm{~h}$, with single 10-min exposure images taken every 11 minutes at 2x2 on-chip binning (effective pixel size $26 \mu \mathrm{m} \times 26 \mu \mathrm{m}$ ).

\section{Image analysis}

The images obtained at the assays were processed with ImageJ (http://imagej.nih.gov/ij/). Bright-field images were adjusted to the best fit for brightness/contrast. For bioluminescence images, a 16-color LUT was applied. Cosmic ray background was removed with the noise>remove outlier function of ImageJ (1 pixel radius, threshold 5). The brightness/contrast was adjusted to enhance dynamic range for visualization of bioluminescence associated with of Gli-Luc expression in each sample, without changing original pixel grey level values. Zero value on LUT scale excludes background. LUT value scales according with these settings are shown in figures.

\section{Results and Discussion}

\section{Gli-Luc-directed bioluminescence correlates with Shh signaling activity in E9.5-E11.5 mouse embryos}

Postnatal $\mathrm{Tg}(\mathrm{Gli}$-Luc) mice display bioluminescence, mostly visualized in the skin, foot pads and possibly in the developing cerebellum, that can be reversibly abolished by Smo antagonists, which supports the notion that bioluminescence is produced as a consequence of $\mathrm{Hh}$ signaling activation (Kimura et al., 2008). In addition, this reportshowed high bioluminescence intensity in E15.5 embryos of this transgenic line, but the corresponding distribution pattern of Gli-related transcriptional activation (i.e., due to the Gli transcriptional activators, GliA, Gli1 and Gli2; Jiang, Hui, 2008) was not determined. To evaluate GliA activity in real time during mouse embryo development, we developed a functional real-time imaging system to visualize cultures of embryos or embryo explants of the Tg(Gli-Luc) mouse line (Fig. 1). Whole E9.5-E11.5 Tg(GliLuc) embryos were recorded for up to 27 hours in order to observe the emergence and extinction of Hh-related bioluminescence in distinct embryo regions.

Bioluminescence intensity in E9.5 embryos was low but detectable and spatially restricted (Fig. 2A and Suppl. Video 1). The presence of Shh, Ptch and/or Gli1/2 has been reported in the posterior region of the developing forelimb, the distal zone of the first branchial arch, the frontonasal prominence, the differentiating somites and the presomitic/somitic mesoderm (Ahn, Joyner, 2004; Hahn et al., 1996; Harfe et al., 2004; Jeong et al., 2004; Marti et al., 1995; McDermott et al., 2005; Platt et al., 1997). For the first branchial arch, the appearance and extinction of bioluminescence in its distal area is likely coincident with the beginning and the end of its outgrowth, a phenomenon regulated by Shh (Jeong et al., 2004), whereas, the emergence of bioluminescence in the posterior area of the developing forelimb reports the initiation of Shh activity associated with the zone of polarizing activity (ZPA) that controls digit specification (see below) (Ahn, Joyner, 2004; Harfe et al., 2004). On the other hand, the strong bioluminescence in the very posterior zone of the embryo is coincident with the progressive somitogenesis still occurring at this stage (McDermott et al., 2005; Platt et al., 1997)

Despite the good correlation described above, the pattern
Fig. 1. Experimental design. (A) Preparation ofTg(Gli-Luc) embryos or embryo explants for bioluminescence visualization during in vitro development. A lateral view of a midgestation Tg(Gli-Luc) mouse embryo is represented (E9.5-E11.5 were used); purple lines indicate the site of cuts performed to dissect the developing midbrain or limbs. The midbrain (a coronal slice is represented) was additionally sliced at the dorsal side (D) to obtain an "open book" conformation, such that the ventral area $(V)$ is at the center, flanked by dorsal areas, and the ventricular surface facing upwards. The embryos, explants or limbs were placed in a Petri dish. Collagen or agarose was used to immobilize developing midbrain explants or embryos, respectively, during culture. Limbs were cultured on a floating filter. A, anterior; P, posterior. (B) Scheme of the equipment employed to capture bioluminescent signal from live embryos or embryo explants. Embryos or embryo explants in a Petri dish were placed within a chamber that provided controlled heat and humidity; $\mathrm{CO}_{2}$ was pumped from a regulator connected to the chamber. The chamber was located over the stage of a stereo microscope and bioluminescence from the sample was captured by an EM-CCD or slow-scan CCD camera attached to the microscope in complete environmental darkness; data were processed by an external computer.

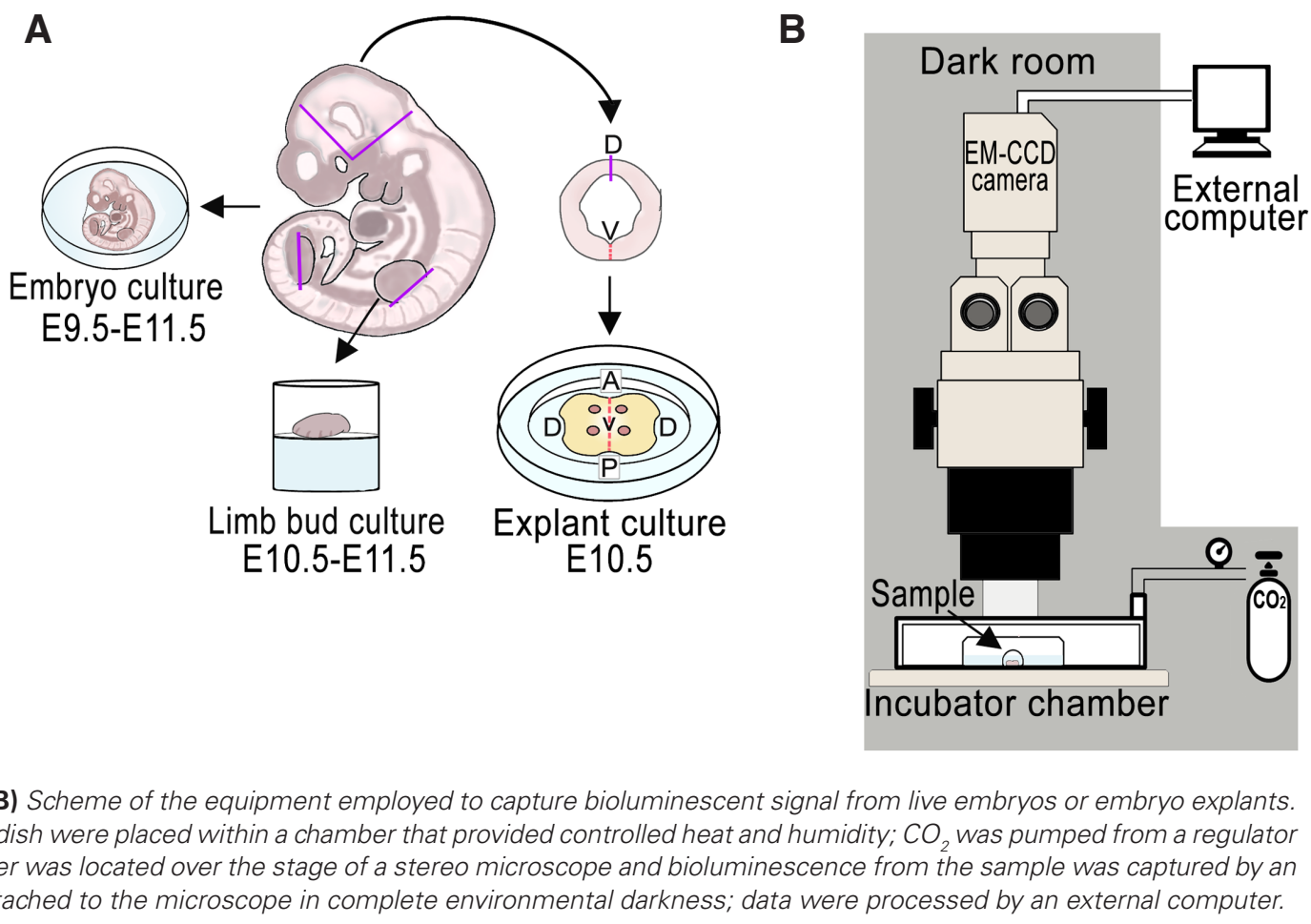


observed in the dorsal area around the midbrain has not been consistently detected using in situ hybridization or immunohistochemistry (see references above), though one report shows the presence of Gli1 mRNA in what appears to be the same domain (Platt et al., 1997), and although Gli2 mRNA is present in this area (Mavromatakis et al., 2011), it has been associated with transcriptional repression rather than activation. In the ventral midbrain, Shh, initially secreted from the notochord (first phase) and then from the floor plate (second phase), specifies progenitors of ventral neurons, including those in the floor plate that give rise to the mesencephalic dopaminergic neurons, and regulates the initial phases of neuronal differentiation (Hayes et al., 2013). The first phase of Shh signaling within the floor plate occurs prior to the developmental stages analyzed in this study, but during the initiation of the second phase at E9.5, GliAactivity was not detected, despite being an area that, at this developmental stage, shows consistent presence of all Hh components (Hayes et al., 2013; Mavromatakis et al., 2011; Platt et al., 1997). On the basis of the bioluminescent

A

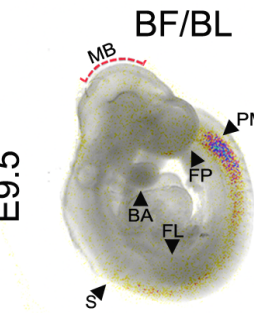

time (h)
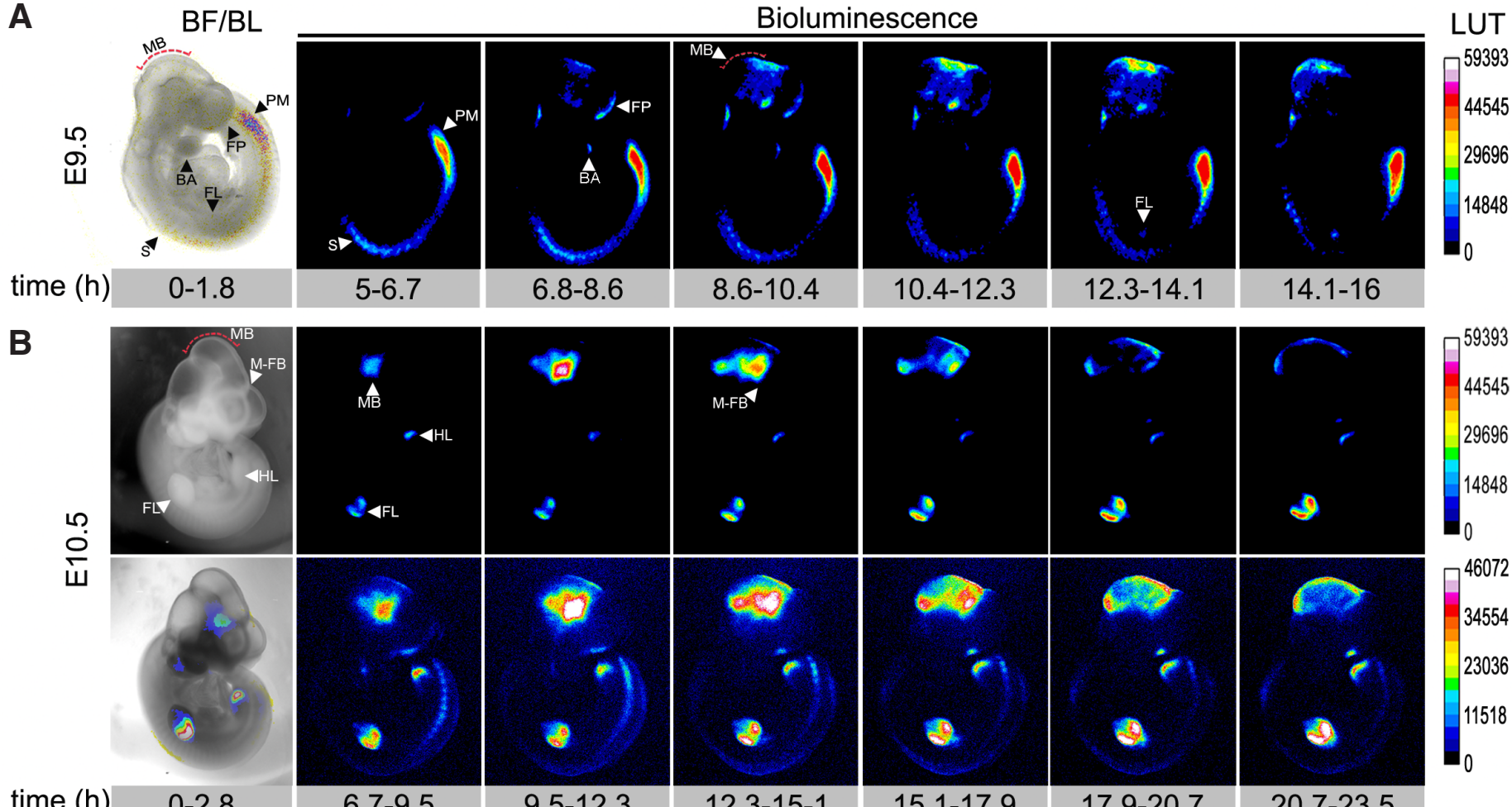

12.3-14.1

$14.1-16$

time (h) $\quad 0-2.8$

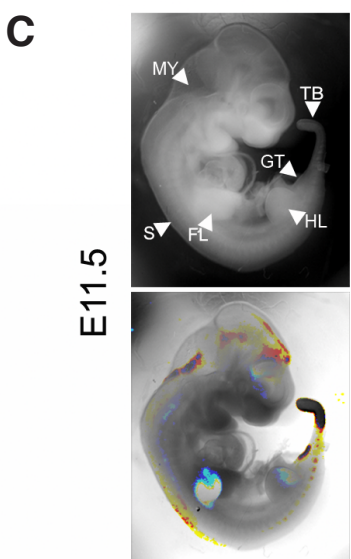

time (h)

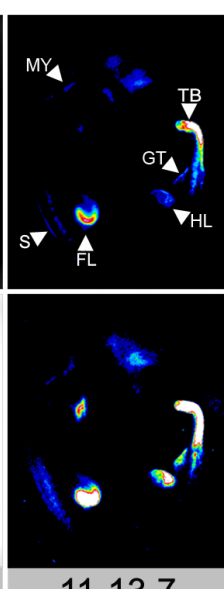

$11-13.7$
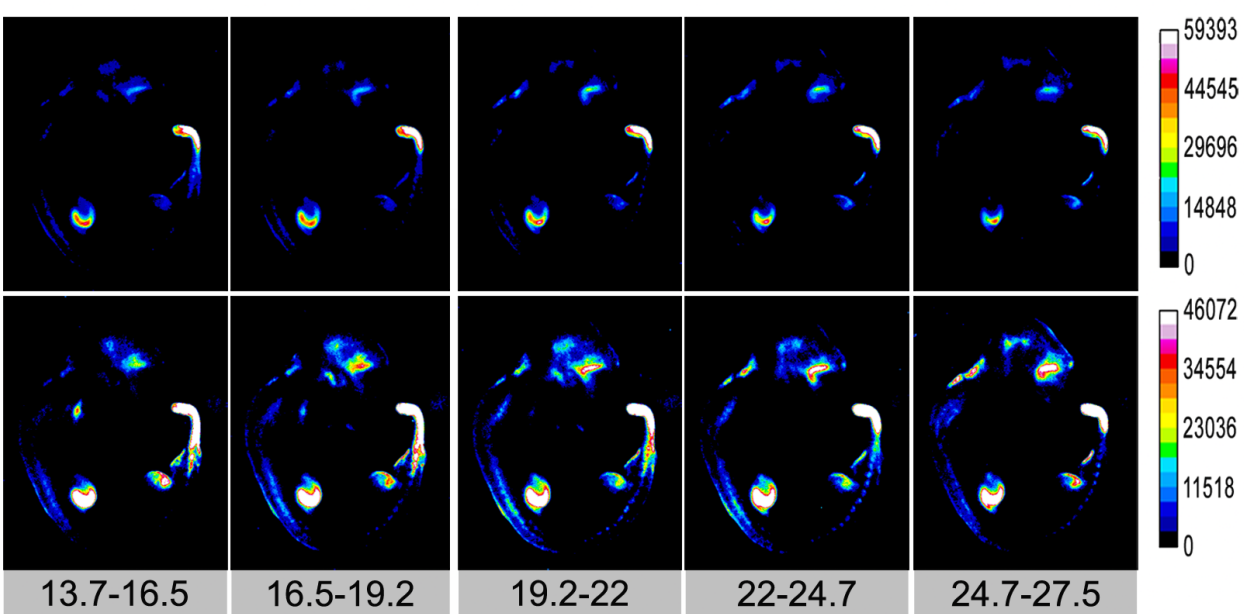

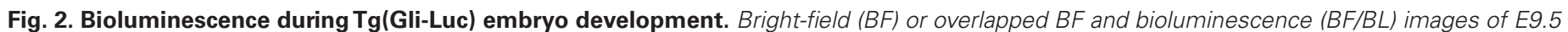

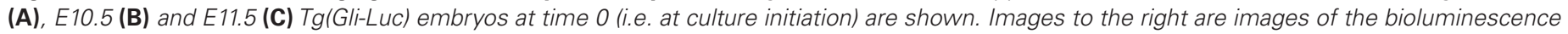

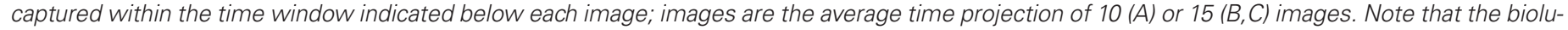

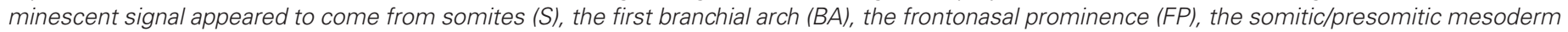

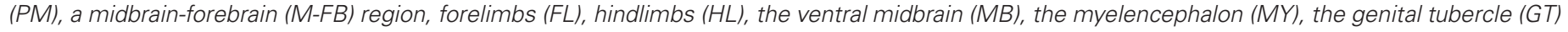

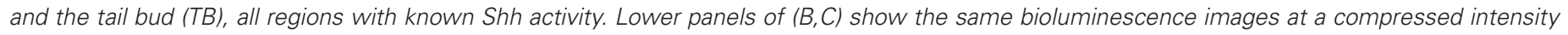
scale. In no case additional sites of bioluminescence emission were found. 
pattern observed in E10.5 embryos (see below), this unexpected observation suggests that the promoter of Gli-Luc only responds to GliA activity either under certain transcriptional genome contexts, including DNA sequences and transcriptional protein complexes surrounding the GliA-responsive elements, or in the presence of relatively high levels of GliA proteins (more discussion regarding this issue below). Due to the proximity of floor plate and somites, at the obtained spatial resolution we cannot discard the possibility

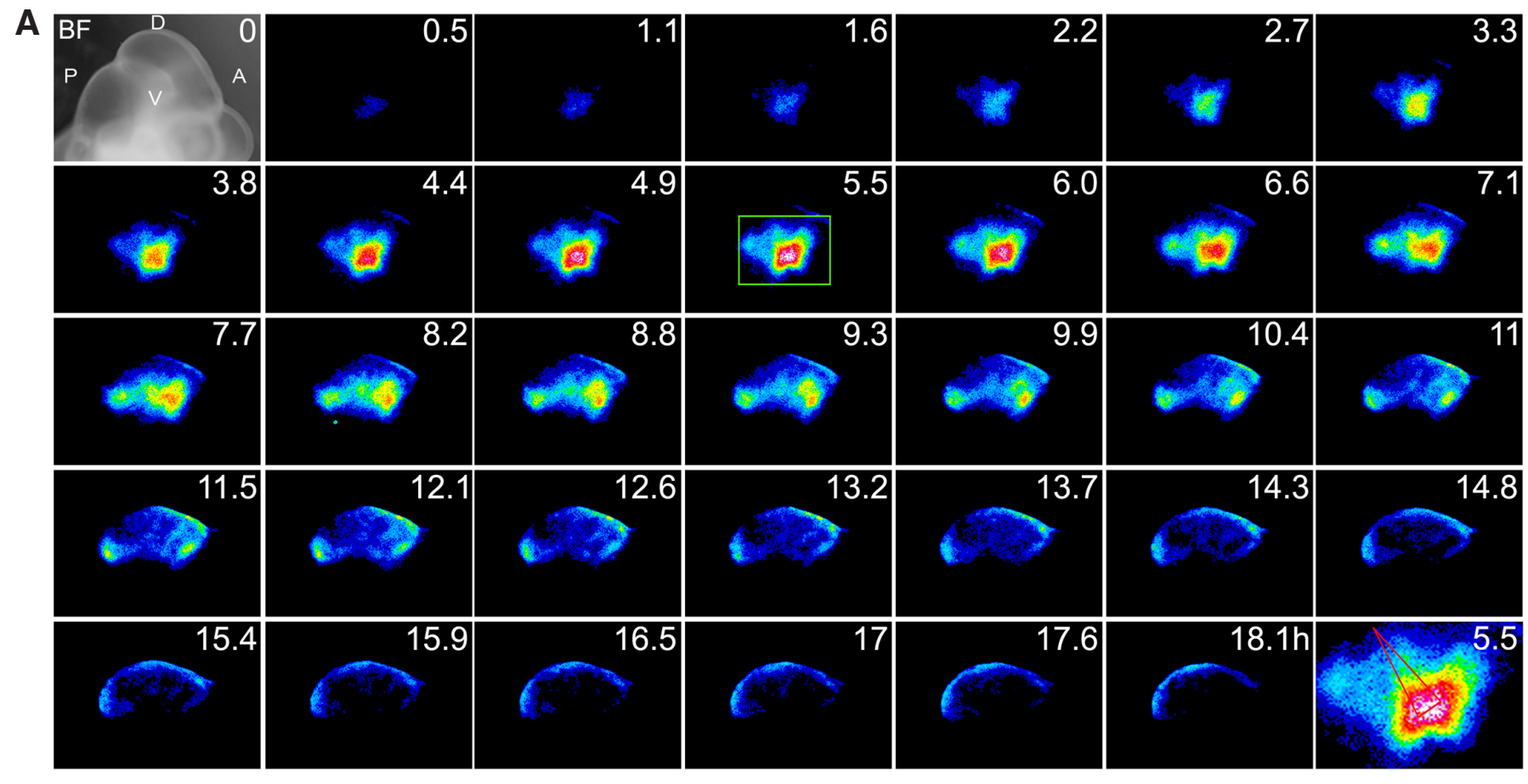

B
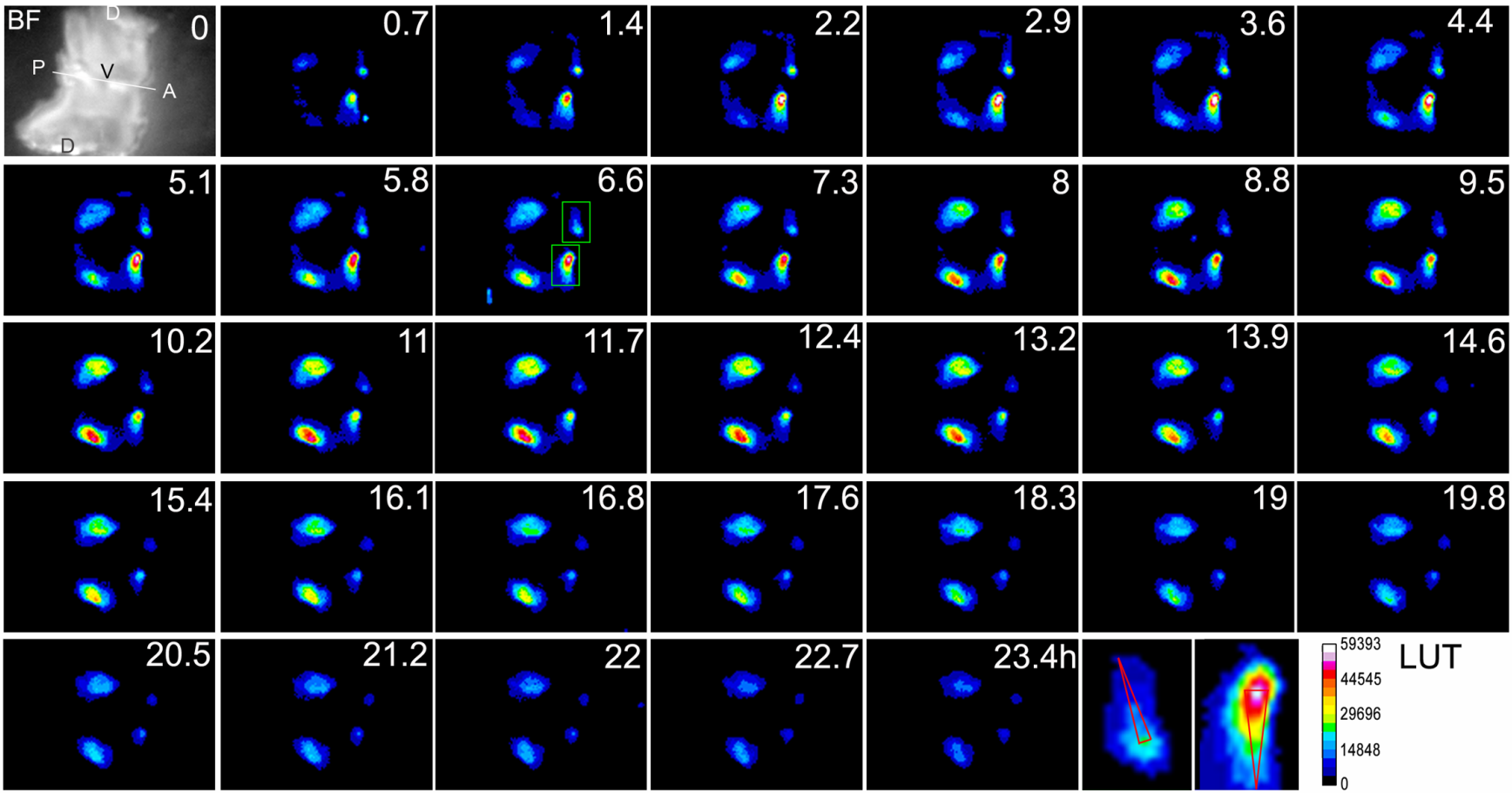

Fig. 3. Bioluminescence imaging in the midbrain during E10.5 Tg(Gli-Luc) embryo or midbrain explant development. (A) The midbrain area of a developing E10.5 Tg(Gli-Luc) embryo was selected for closer analysis. Each image is a projection of the time-average of 3 individual images. Note that the signal increased first in an anterior (A) domain and then emerged from a posterior (P) domain. (B) Midbrain explants from E10.5Tg(Gli-Luc) embryos were cultured for up to $24 \mathrm{~h}$, the period during which bioluminescence was determined. Each image represents the time-average of 4 individual images. Bioluminescence was emitted in restricted domains that emerged with a similar pattern to bioluminescent domains in the intact embryo, though absolute bioluminescence intensity was at a lower range. Red triangles in $(A, B)$ represent the signal gradient in magnifications of the area enclosed in green squares. 
that bioluminescence is also absent along the developing spinal cord, where Shh in the floor plate specifies neighboring domains that give rise to motoneurons, among other neuron types (Martí, Bovolenta, 2002).

At E10.5, the bioluminescence remained associated with areas corresponding to differentiating somites where expression of genes encoding Hh components have been reported, as mentioned above, and only extended to few other areas, even when the signal was overexposed (Fig. 2B and Suppl. Video 2). Notably, the ventral area of the midbrain and the ZPA of developing limbs reported relatively high levels of bioluminescence when compared to those detected in E9.5 embryos or other regions of E10.5 embryos. Shh activity has been studied in detail in these two areas (Ahn, Joyner, 2004; Harfe et al., 2004; Hayes et al., 2013). Note that at this stage the posterior region of hindlimbs are beginning to show GliA activity, in agreement with their delayed development in comparison with forelimbs.

At E11.5, elevated levels of bioluminescence remained in the developing limbs, evident in forelimbs but also in hindlimbs, and emerged in the genital tubercle and in the tail bud where Shh activity has been reported (Haraguchi et al., 2001; Fig. 2C and Suppl. Video 3). At this stage, bioluminescence was still detected in what appears to be the posterior and anterior edges of mesencephalon, myelencephalon and in somites.

To corroborate that the bioluminescent signal detected in embryos described above is Shh-dependent (Fig. 2 A,B), E9.5 and E10.5 embryos were cultured in the presence or absence of the Hh signaling inhibitor cyclopamine (Fig. 5A). Notably near all the
A BF

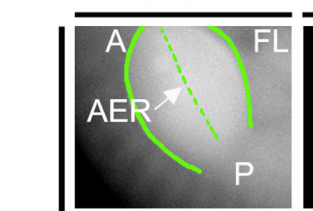

P
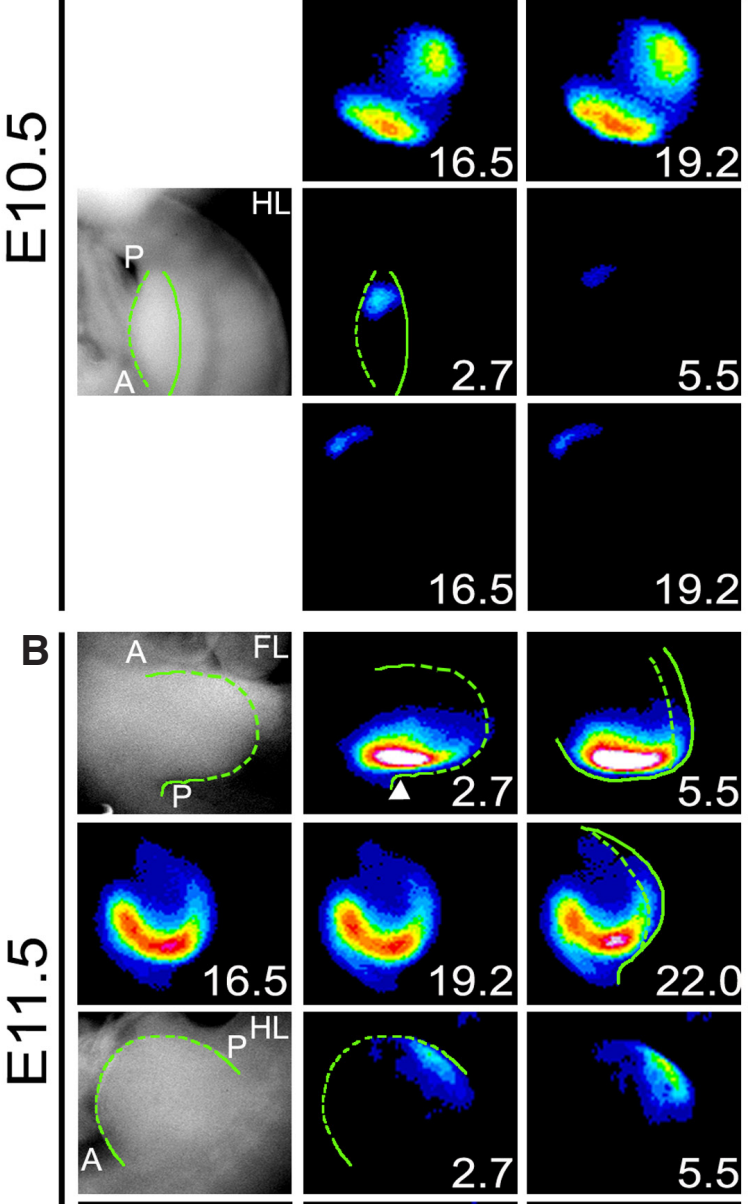

L

ए
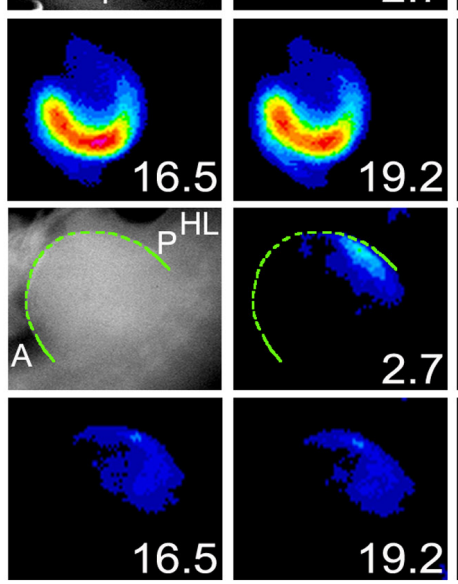

Bioluminescence
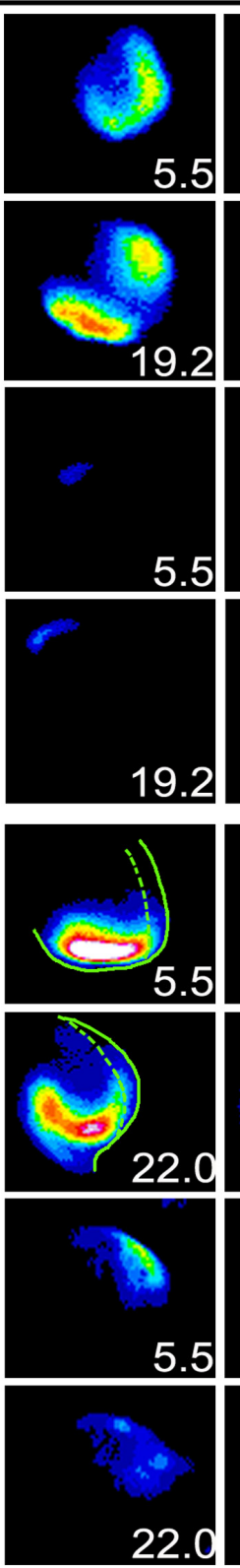
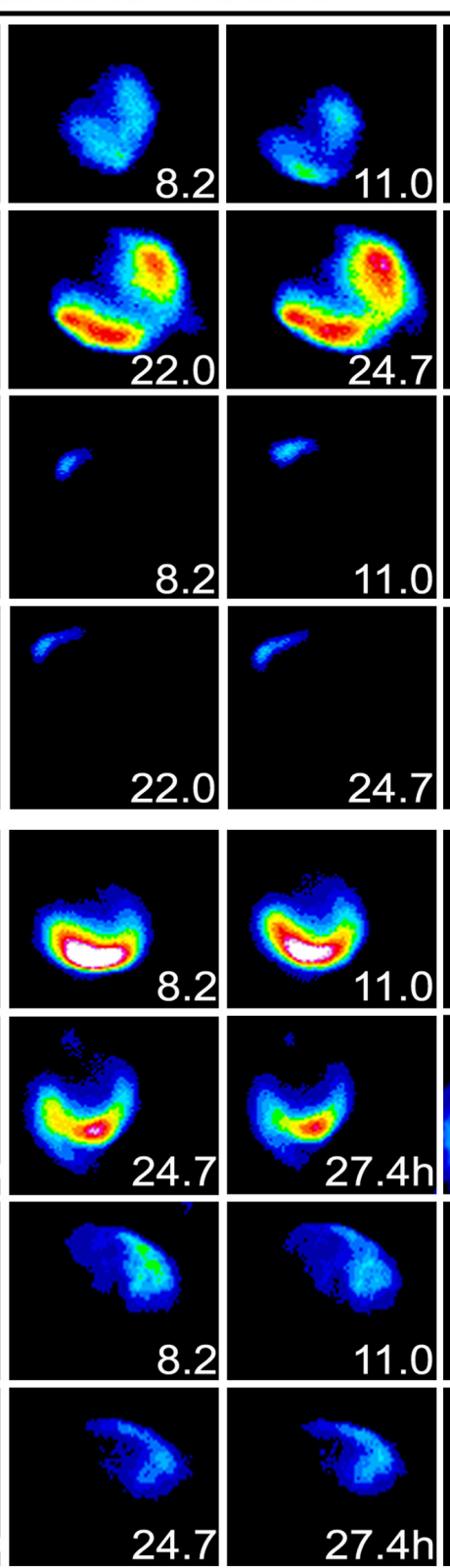
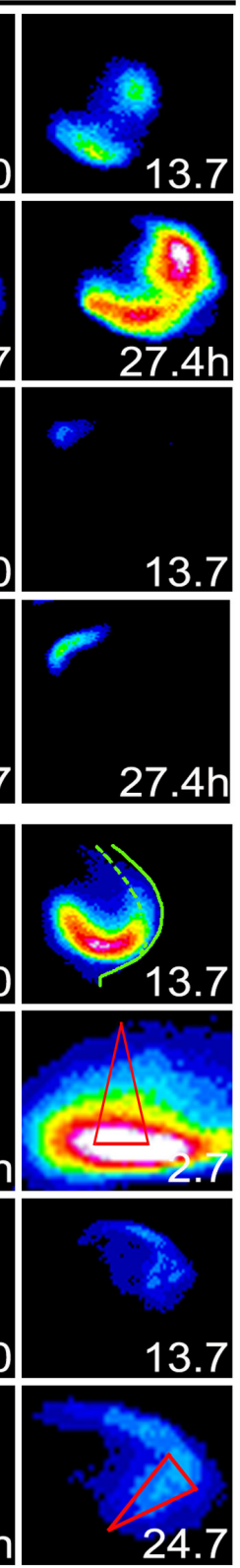

LUT

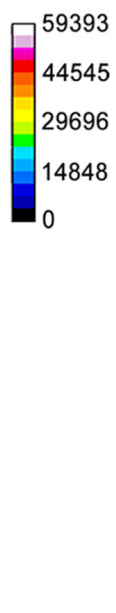

Fig. 4. Bioluminescence imaging in developing limbs of Tg(Gli-Luc) embryos. The forelimb (FL) and the hind limb (HL) area of developing E10.5(A) and E11.5 (B) Tg(Gli-LuC) embryos was selected for closer analysis of changes in bioluminescence pattern and intensity. Each image is the projected temporal average of 15 images. Note that bioluminescence emerged at a posterior domain and divides into dorsal and ventral subdomains that increased in intensity over time. In addition, a posterior to anterior spread of bioluminescence was obvious, suggesting that a signaling gradient was being detected. The green line depicts relevant contours of the limb detected in bright-field (BF) images. AER, apical ectodermal ridge (dotted green line); $A$, anterior; P, posterior. Red triangles represent the signal gradient at the indicated time. 
A

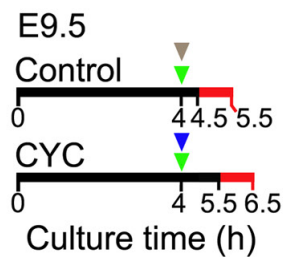

E10.5

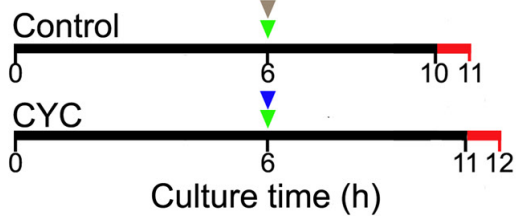

Control CYC V 1h record $=$

E9.5
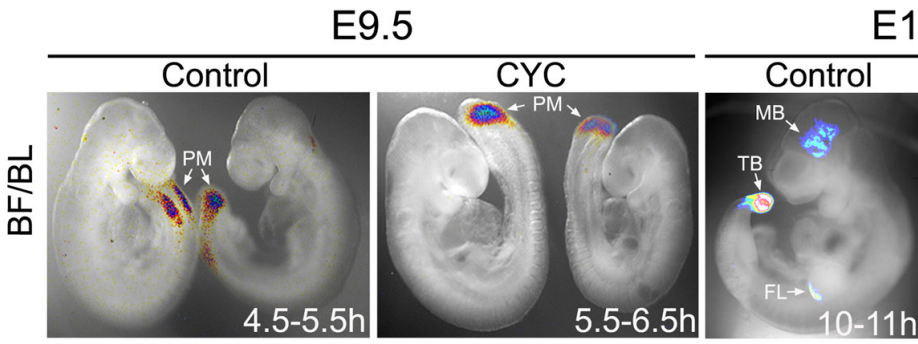

E10.5
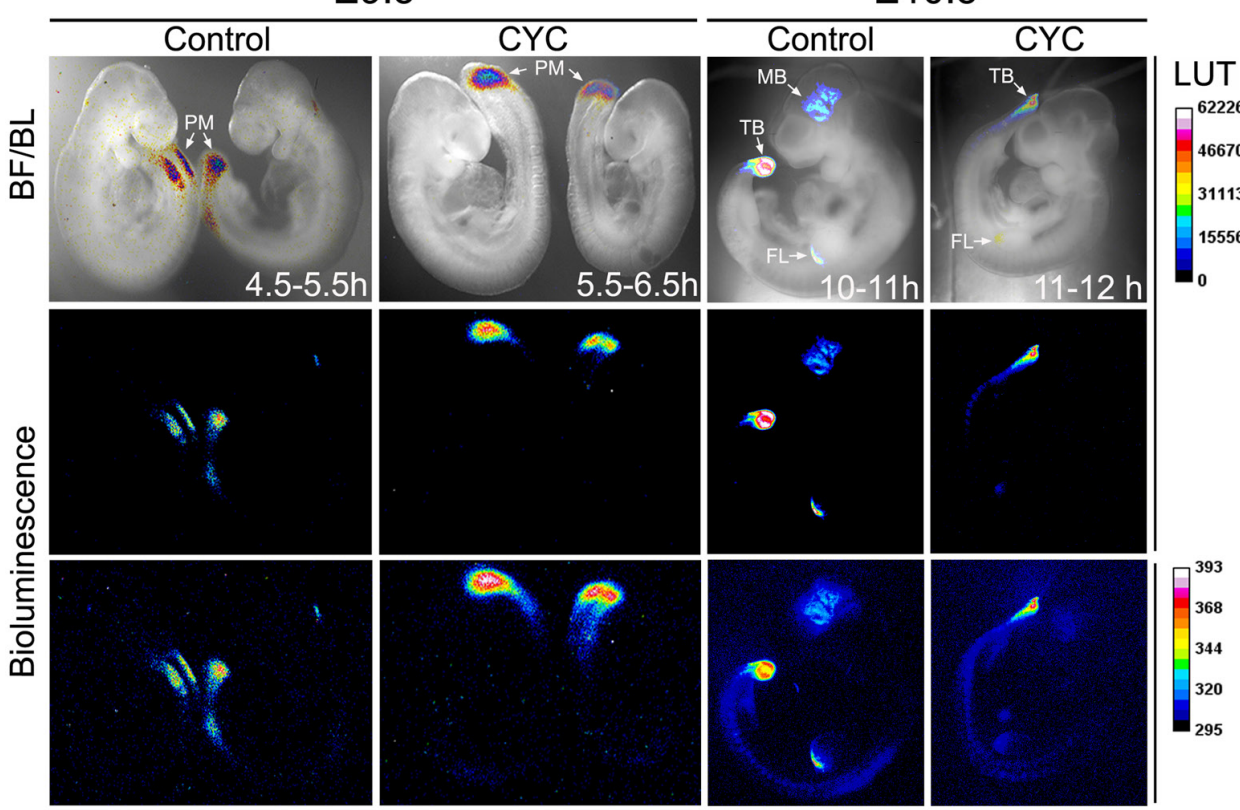

B

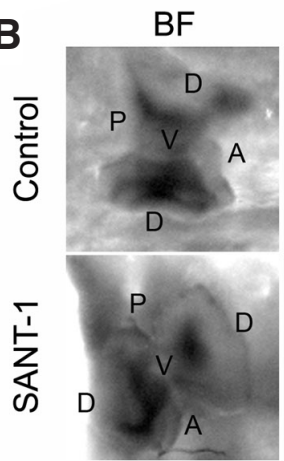

$\mathrm{Oh}$
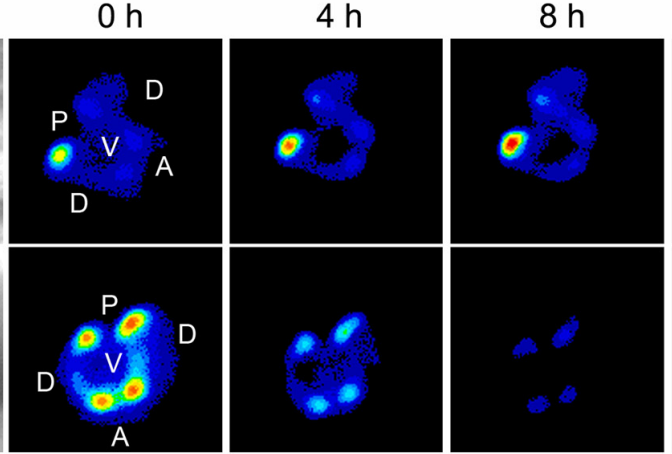

C

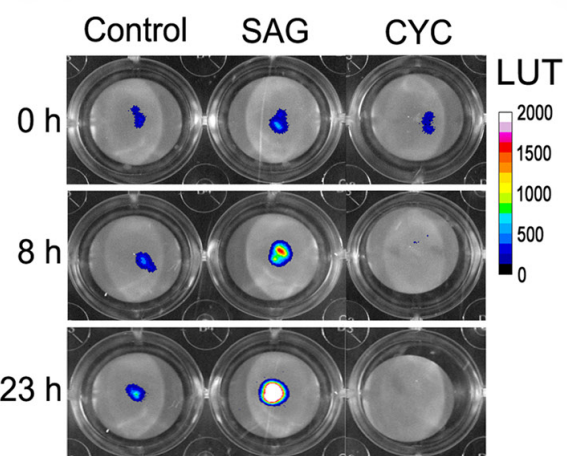

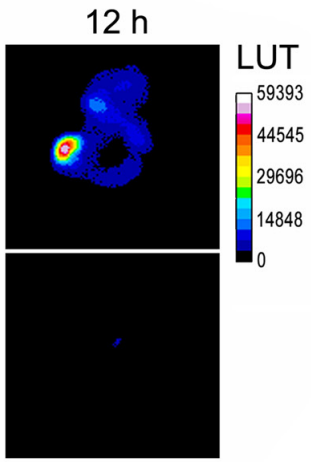

$\square^{393}$

368 344

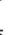
anterior area of the midbrain, in which the signal progressively increased and then mildly extended posteriorly, mainly within the ventral region (Fig. $3 \mathrm{~A}$ and Suppl. Video 4). As bioluminescence

Fig. 5. Bioluminescence is Shh-dependent in embryos and in cultured midbrain explants and limbs. (A) E9.5 or E10.5 embryos were cultured in the presence or absence of cyclopamine (CYC) according with the protocol depicted in diagram lines; note that although Control and CYC samples were treated in parallel, there was a $30 \mathrm{~min}$ lag in bioluminescence determination between both groups. Overlapped bright-field and bioluminescence (BF/BL; top row) and bioluminescence (middle row) images of E9.5 and E10.5 Tg(Gli-Luc) embryos treated with DMSO (Control) or with cyclopamine (CYC) and recorded for the indicated time are shown; bottom row images are at a compressed intensity scale. All images are the average time projection of 6 images. (B) Midbrains of homozygous Tg(Gli-Luc) were cultured in the presence or absence of SANT-1, another Hh signaling inhibitor as $C Y C$, and bioluminescence images taken at the time indicated ( $A$, anterior; $P$, posterior; $D$, dorsal; $V$, ventral). (C,D) Dissected limbs of E10.5 Tg(Gli-Luc) embryos were treated

with DMSO (Control), the Hh agonist SAG and CYC and bioluminescence images (overlapped with BF images) captured with the In-Vivo Xtreme instrument at the time indicated (C) or recorded for $6 \mathrm{~h}$ with the system established here ( $\mathrm{D}$; arrowheads, ZPA). Note the extinction of bioluminescence in samples treated with CYC or SANT-1 and the continuous increase in bioluminescence, for up to 23 , in limbs treated with SAG. 
signal decreased in the anterior area, it is apparent that another bioluminescence focus emerged in the posterior ventral midbrain, which also progressively increased in intensity and then disappeared at nearly the same time as the signal in the anterior region (Fig. 3A and Suppl. Video 4). To further examine the above pattern, GliA activity-related bioluminescence was followed during mesencephalic neural differentiation in explants of E10.5 midbrain. Explant cultures could not reproduce the bioluminescence intensity observed in whole embryos, which emerged and disappeared at a much slower rate (12 vs. 24 h) (Fig. 3B and Suppl. Video 5). However, as expected, GliA activity emerged lateral to the midline with a bioluminescence pattern similar to that observed in whole embryos. In particular, it was evident that GliA activity emerged earlier in the anterior than in the posterior region as independent activity foci; note that these bioluminescent foci were ablated by the Hh antagonist SANT-1 (Fig. 5B). It is relevant to mention that the mRNA of Shh and its Ptch receptor show an apparent homogeneous distribution along the ventral midbrain (Jeong et al., 2004; Platt et al., 1997); however, the observations presented here and the location of Gli1 mRNA indicate that strong Shh signaling is restricted to anterior and posterior midbrain domains. In addition, the graded ventral to dorsal spread of bioluminescence from the anterior domain suggests that a signaling gradient is being formed (Fig. 3 A,B). This strong Shh signaling determined by the Gli-LuC reporter at E10.5 stage was not associated with the specification of dopaminergic neurons of the substantia nigra that occurs at an earlier stage and could only be part of the Shh signal that contributes to the specification of a set of ventral tegmental area dopaminergic neurons and of other neurons and astrocytes derived from more lateral ventral domains (e.g., Nkx2.2 and Nkx6.1; (Blaess et al., 2011; Hayes et al., 2011)).

\section{High bioluminescent intensity in developing limbs represents the strong Shh signaling activity of the zone of polarizing activity}

The other structure where we detected relatively high GliA activity was the developing limb. Here, Shh signaling emerged from the ZPA, where a morphogenetic function gives identity to digits (Harfe et al., 2004). Although it was accepted for many years that a morphogen concentration gradient generated from the ZPA source was the mechanism for digit specification, a study based on genetic manipulations suggests that the time of exposure to Shh is also relevant for the specification of digit mesenchymal cells (Ahn, Joyner, 2004; Harfe et al., 2004). Following bioluminescence in developing fore- and hind-limbs of E10.5 and E11.5 Tg(Gli-Luc) embryos, Shh signaling was observed to emerge within the ZPA domain as a single spot which subsequently divided into two foci, one ventral and one dorsal, whereby bioluminescence increased locally and signal progressively spread anteriorly (Fig. 4 A,B and Suppl. Video 6-9). The gradual spatial and local increase in bioluminescence could be indicative of the active development of the expected Shh signaling gradient that determines digit specification. Considering the highest and lowest values of bioluminescence intensity detected, the dynamic range of the Gli-Luc reporter in limbs of Tg(Gli-Luc) embryos is of up to 10-fold signaling difference. All bioluminescence detected in limbs is Shh-dependent as a marked decrease in bioluminescence occurred in the presence of cyclopamine (a Hh signaling inhibitor) whereas a converse increase in the posterior region and the apparent emergence in the anterior region was observed following treatment with SAG (a Hh signaling agonist) in cultured limbs (Fig. 5 C,D).

\section{Concluding remarks}

The present study shows that the $\mathrm{Tg}$ (Gli-Luc) transgenic mouse line is a useful model to follow Shh activity in real time during embryogenesis. However, it is apparent that the capacity of the Gli-Luc reporter to report Shh activity is context-dependent and may be restricted to relatively high signaling activation. This latter condition likely correlates with the time at which the high levels of GliA are sufficient to activate Gli-Luc transcription, but it could also be associated with low levels of GliR, such as Gli3, which commonly accompanies Shh signaling during development (Büscher, Ruther, 1998; Mavromatakis et al., 2011). The genomic context of GBS can also determine differential responsiveness to Shh signaling. For instance, different sets of genes are regulated by Gli in different developing limb domains, which could relate to variations in the GBS but also appears to result from the genomic context surrounding such binding sequences (Lewandowski et al., 2015). Cooperative binding of transcription factors is a common mechanism controlling gene activation that, if required for GliAmediated transcriptional activation in a specific spatiotemporal context, would not be detected by the Gli-Luc reporter used here. For instance, in the ventral midbrain of E9.5 embryos, although the inability to detect bioluminescence was possibly due to the repressive action of Gli2/Gli3 present in this area (Mavromatakis et al., 2011), transcriptional activation might also depend on the cooperative binding of GliA with SoxB1 neural associated transcription factors within the regulatory sequences of relevant target genes (Oosterveen et al., 2012).

In addition to the excellent correlation between areas of observed bioluminescence and known Shh activity in Tg(Gli-Luc) embryos, the Gli-Luc reporter also displayed a wide dynamic range detecting up to 10-fold difference in bioluminescence intensity. Presently, the poor quantitative power of regular in situ hybridization or immunohistochemistry protocols used in most studies reporting the presence Shh signaling molecules in embryos, and the unknown transcription factor interactions required in some developmental context, as referred to above, impose limitations for any correlation between bioluminescence intensity due to Gli-Luc expression and Shh signaling levels. However, even under this panorama, it was interesting to observe that in many instances the bioluminescent signal due to Gli-Luc expression was displayed as foci that correspond to sources of Shh protein (Marti et al., 1995), that emerge and extinguish themselves with time and, thus, possibly correspond to signaling centers. The dynamics of these signaling centers was particularly evident in the limb (Fig. 4B and Suppl. Video 8-9) where a spatial gradient could be detected and, in addition, the local graded increase in bioluminescence within the ZPA opens the possibility of a diffusion-independent $\mathrm{Hh}$ signaling gradient that evolves with time and might be relevant for digit specification. This implies that Hh signaling level should be added to the time of signaling activity as part of the mechanism by which Shh contributes to the determination of cell identity (Harfe et al., 2004). Although the pattern of bioluminescent foci observed could be coincident with the temporal emergence of centers of high Shh activity (i.e., containing high levels of GliA), the initiation of Hh signaling might not be detected. This could result from the inability of the Gli-Luc 
reporter to detect low levels of GliA, or the requirement for cooperation with other transcription factors at this stage (Oosterveen et al., 2012). Consistent with this possibility, the transcriptional activity of Gli2, the initial effector of the Hh signaling pathway in several developmental contexts (Bai et al., 2002) is less efficiently detected by the Gli-Luc reporter used here (Sasaki et al., 1999).

Detection of cell signaling by following gene activation in real time is an invaluable tool for the molecular understanding of development. As shown here even the use of a simple promoter containing tandem repeats of the binding sequence for a single transcription factor is capable of reporting the dynamics of cell signaling. At least for Shh, coupling novel and more efficient luciferases (e.g., Akaluc; Iwano et al., 2018) with promoters retaining the natural gene context (e.g., that of Ptch1; Kogerman et al., 1999) will greatly facilitate the analysis of the complex cell signaling dynamics associated with this key morphogen.

\section{Acknowledgments}

We thank Gilda Guerrero-Flores, Aimée Bastidas-Ponce and Graciela Cabeza for their technical assistance. This work was supported by DGAPA/ UNAM (PAPIIT grants IN223810, IA200915, IN213416 and IA202317) and CONACYT grants 239891,132478 and FOINS1723. We thank Dr. Tom Curran for the donation of the Fvb/NJ Tg(Gli-Luc) transgenic mouse line.

\section{References}

AHN S, JOYNER AL (2004). Dynamic Changes in the Response of Cells to Positive Hedgehog Signaling during Mouse Limb Patterning. Cell 118: 505-516.

BAI CB, AUERBACH W, LEE JS, STEPHEN D, JOYNER AL (2002). Gli2, but not Gli1, is required for initial Shh signaling and ectopic activation of the Shh pathway. Development 129: 4753-4761.

BAIZABAL J-M, COVARRUBIAS L (2009). The embryonic midbrain directs neurona specification of embryonic stem cells at early stages of differentiation. Dev. Biol. 325: 49-59.

BLAESS S, BODEA GO, KABANOVAA, CHANET S, MUGNIERY E, DEROUICHEA, STEPHEN D, JOYNERAL (2011a). Temporal-spatial changes in Sonic Hedgehog expression and signaling reveal different potentials of ventral mesencephalic progenitors to populate distinct ventral midbrain nuclei. Neural Dev 6: 29.

BÜSCHER D, RUTHER U (1998). Expression profile of Gli family members and Shh in normal and mutant mouse limb development. Dev Dyn 211: 88-96.

HAHN H, CHRISTIANSEN J, WICKING C, ZAPHIROPOULOS PG, CHIDAMBARAM A, GERRARD B, VORECHOVSKY I, BALE AE, TOFTGARD R, DEAN M, WAINWRIGHT B (1996). A mammalian patched homolog is expressed in target tissues of sonic hedgehog and maps to a region associated with developmental abnormalities. J Biol Chem 271: 12125-12128.

HARAGUCHI R, MO R, HUI C, MOTOYAMAJ, MAKINO S, SHIROISHIT, GAFFIELD W, YAMADA G (2001). Unique functions of Sonic hedgehog signaling during external genitalia development. Development 128: 4241-4250.

HARFE BD, SCHERZ PJ, NISSIM S, TIAN H, MCMAHON AP, TABIN CJ (2004). Evidence for an Expansion-Based Temporal Shh Gradient in Specifying Vertebrate Digit Identities. Cell 118: 517-528.

HAYES L, RALLS S, WANG H, AHN S (2013). Duration of Shh signaling contributes to mDA neuron diversity. Dev. Biol. 374: 115-126.

HAYES L, ZHANG Z, ALBERT P, ZERVAS M, AHN S (2011). Timing of Sonic hedgehog and Gli1 expression segregates midbrain dopamine neurons. J Comp Neurol
519: 3001-3018

HERNÁNDEZ-MARTÍNEZ R, CASTRO-OBREGÓN S, COVARRUBIAS L (2009). Progressive interdigital cell death: regulation by the antagonistic interaction between fibroblast growth factor 8 and retinoic acid. Development 136: 3669-3678.

IWANO S, SUGIYAMA M, HAMA H, WATAKABE A, HASEGAWA N, KUCHIMARU T, TANAKA KZ, TAKAHASHI M, ISHIDAY, HATAJ, et al., (2018). Single-cell bioluminescence imaging of deep tissue in freely moving animals. Science 359: 935-939.

JEONG J, MAO J, TENZEN T, KOTTMANN AH, MCMAHON AP (2004). Hedgehog signaling in the neural crest cells regulates the patterning and growth of facial primordia. Genes Dev. 18: 937-951.

JIANG J, HUI C-C (2008). Hedgehog Signaling in Development and Cancer. Dev. Cell 15: 801-812.

KIMURA H, NG JMY, CURRAN T (2008). Transient Inhibition of the Hedgehog Pathway in Young Mice Causes Permanent Defects in Bone Structure. Cancer Cell 13: 249-260.

KOGERMAN P, GRIMM T, KOGERMAN L, KRAUSE D, UNDÉN AB, SANDSTEDT B, TOFTGARD R, ZAPHIROPOULOS PG (1999). Mammalian suppressor-offused modulates nuclear-cytoplasmic shuttling of Gli-1. Nat Cell Biol 1: 312-319.

LEWANDOWSKIJP, DU F, ZHANG S, POWELLMB, FALKENSTEIN KN, JIH, VOKES SA (2015). Spatiotemporal regulation of GLI target genes in the mammalian limb bud. Dev. Biol. 406: 92-103.

MARTI E, TAKADA R, BUMCROT DA, SASAKI H, MCMAHON AP (1995). Distribution of Sonic hedgehog peptides in the developing chick and mouse embryo. Development 121: 2537-2547.

MARTí E, BOVOLENTA P (2002). Sonic hedgehog in CNS development: one signal, multiple outputs. Trends Neurosci. 25: 89-96.

MAVROMATAKIS YE, LIN W, METZAKOPIAN E, FERRI ALM, YAN CH, SASAK H, WHISETT J, ANG S-L (2011). Foxa1 and Foxa2 positively and negatively regulate Shh signalling to specify ventral midbrain progenitor identity. Mech. Dev. 128: 90-103.

MCDERMOTT A, GUSTAFSSON M, ELSAM T, HUI C-C, EMERSON CP, BORYCKI A-G (2005). Gli2 and Gli3 have redundant and context-dependent function in skeletal muscle formation. Development 132: 345-357.

OOSTERVEENT, KURDIJAS, ALEKSEENKOZ, UHDE CW, BERGSLAND M, SANDBERG M, ANDERSSON E, DIAS JM, MUHR J, ERICSON J (2012). Mechanistic Differences in the Transcriptional Interpretation of Local and Long-Range Shh Morphogen Signaling. Dev. Cell 23: 1006-1019.

PIETROBONO S, GAGLIARDI S, STECCA B (2019). Non-canonical Hedgehog Signaling Pathway in Cancer: Activation of GLI Transcription Factors Beyond Smoothened. Frontiers Genet. 10: 556

PLATT KA, MICHAUD J, JOYNER AL (1997). Expression of the mouse Gli and Ptc genes is adjacent to embryonic sources of hedgehog signals suggesting a conservation of pathways between flies and mice. Mech. Dev. 62: 121-135.

RIBES V, BALASKAS N, SASAI N, CRUZ C, DESSAUD E, CAYUSO J, TOZER S, YANG LL, NOVITCH B, MARTI E, BRISCOE J (2010). Distinct Sonic Hedgehog signaling dynamics specify floor plate and ventral neuronal progenitors in the vertebrate neural tube. Genes Dev. 24: 1186-1200

SAITO K, CHANG Y-F, HORIKAWA K, HATSUGAI N, HIGUCHI Y, HASHIDA M, YOSHIDA Y, MATSUDA T, ARAI Y, NAGAI T (2012). Luminescent proteins for high-speed single-cell and whole-body imaging. Nature Comm. 3: 1262.

SASAKI H, NISHIZAKI Y, HUI C, NAKAFUKU M, KONDOH H (1999). Regulation of Gli2 and Gli3 activities by an amino-terminal repression domain: implication of Gli2 and Gli3 as primary mediators of Shh signaling. Development 126: 3915-3924.

THORNE N, INGLESE J, AULD DS (2010). Illuminating Insights into Firefly Luciferase and Other Bioluminescent Reporters Used in Chemical Biology. Chem. Biol. 17: 646-657. 


\section{Further Related Reading, published previously in the Int. J. Dev. Biol.}

Role of Hox genes in regulating digit patterning

Rocío Pérez-Gómez, Endika Haro, Marc Fernández-Guerrero, María F. Bastida and María A. Ros

Int. J. Dev. Biol. (2018) 62: 797-805

https://doi.org/10.1387/ijdb.180200mr

Sonic hedgehog in vertebrate neural tube development

Marysia Placzek and James Briscoe

Int. J. Dev. Biol. (2018) 62: 225-234

https://doi.org/10.1387/ijdb.170293jb

Temporal and spatial expression patterns of Hedgehog receptors in the developing inner and middle ear

Jeong-Oh Shin, Harinarayana Ankamreddy, Naga Mahesh Jakka, Seokwon Lee, Un-Kyung Kim and Jinwoong Bok

Int. J. Dev. Biol. (2017) 61: 557-563

https://doi.org/10.1387/ijdb.170155jb

Unusual pattern of Sonic hedgehog expression in the polydactylous mouse mutant Hemimelic extra-toes

Isabelle Blanc, Antoine Bach and Benoît Robert

Int. J. Dev. Biol. (2002) 46: 969-974

http://www.intjdevbiol.com/web/paper/12455636

Sonic hedgehog: a common signal for ventral patterning along the rostrocaudal axis of the neural tube.

J Ericson, J Muhr, T M Jessell and T Edlund

Int. J. Dev. Biol. (1995) 39: 809-816
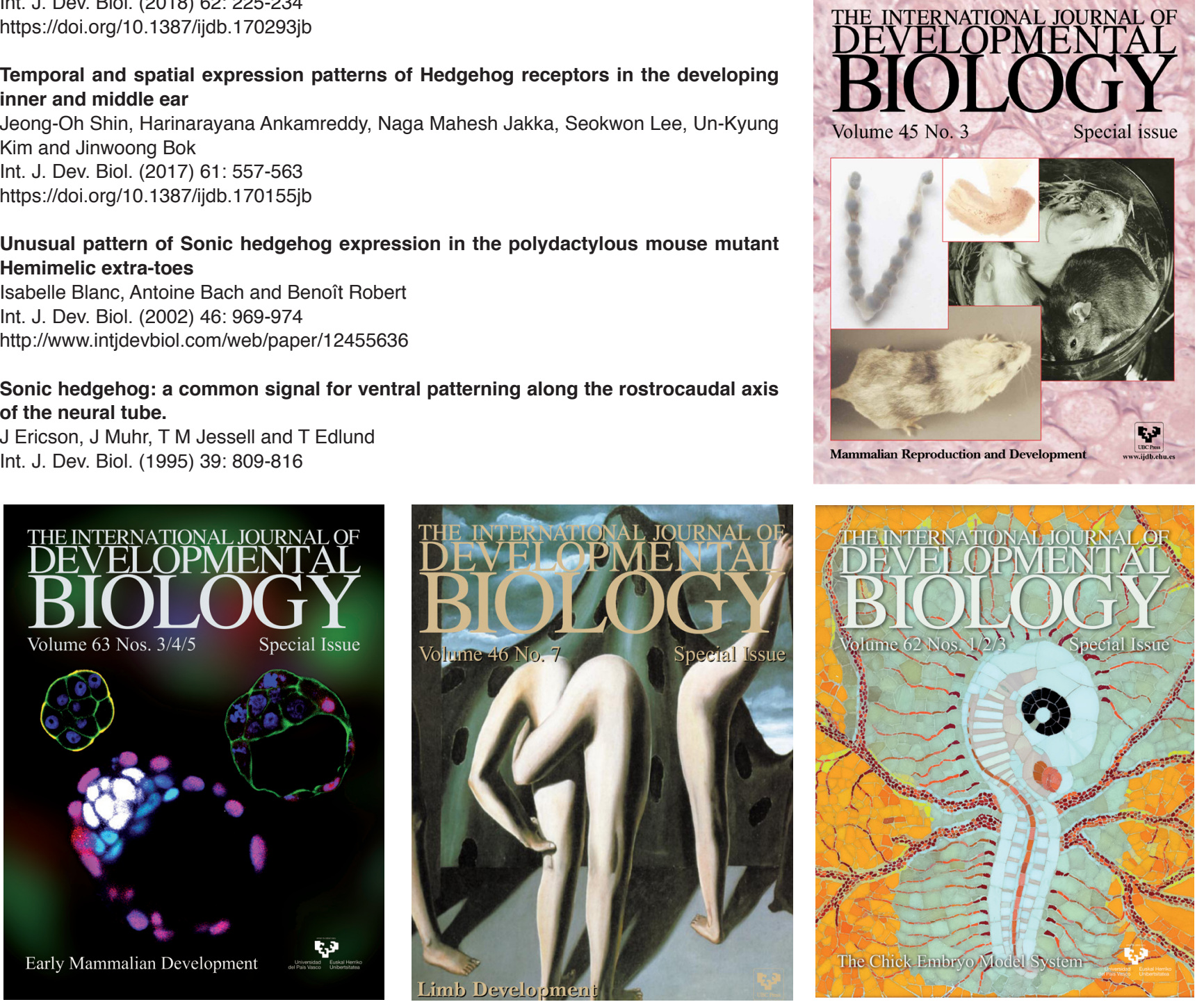